\title{
Sound Velocities in Shocked Liquid Deuterium
}

\author{
N.C. Holmes \\ W.J. Nellis \\ M. Ross
}

This paper was prepared for submittal to the

Shock Wave Conference

Amherst, MA

July 27-August 1, 1997

August 1997

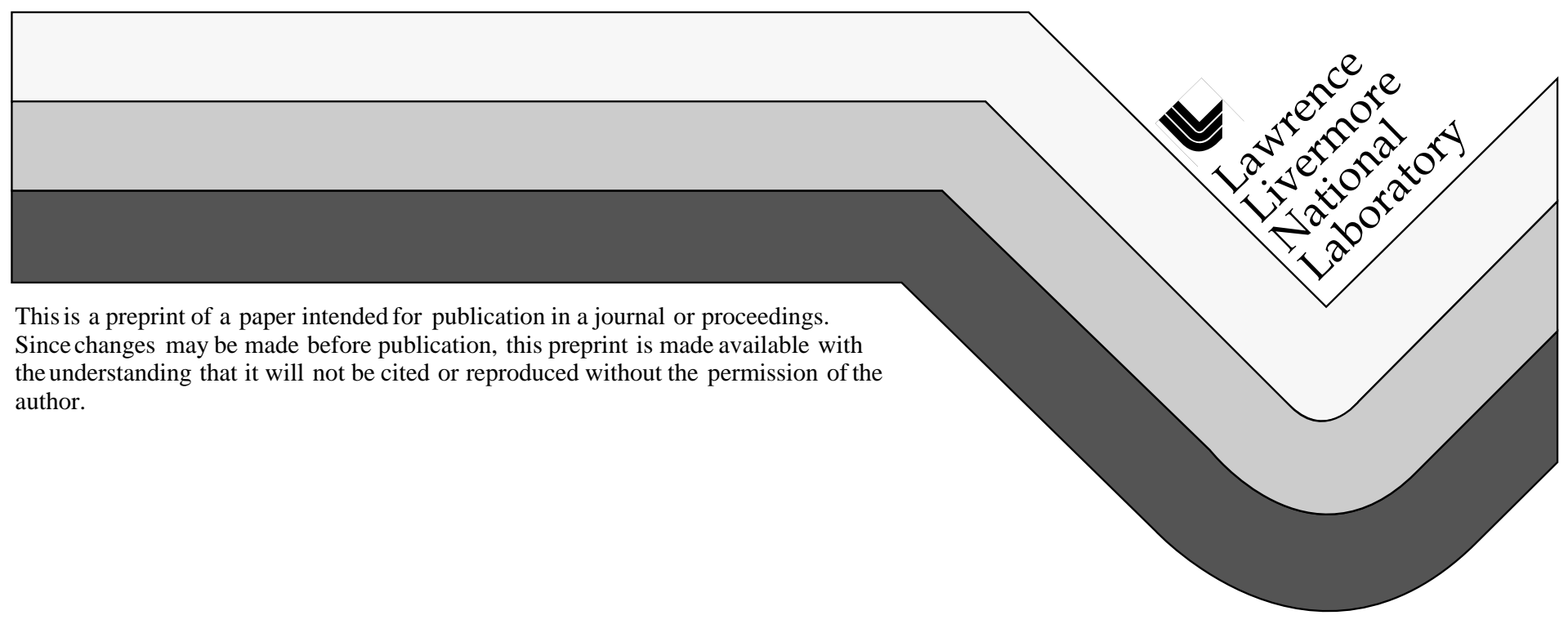




\section{DISCLAIMER}

This document was prepared as an account of work sponsored by an agency of the United States Government. Neither the United States Government nor the University of California nor any of their employees, makes any warranty, express or implied, or assumes any legal liability or responsibility for the accuracy, completeness, or usefulness of any information, apparatus, product, or process

disclosed, or represents that its use would not infringe privately owned rights. Reference herein to any specific commercial product, process, or service by trade name, trademark, manufacturer, or otherwise, does not necessarily constitute or imply its endorsement, recommendation, or favoring by the United States Government or the University of California. The views and opinions of authors expressed herein do not necessarily state or reflect those of the United States Government or the University of California, and shall not be used for advertising or product endorsement purposes. 


\title{
SOUND VELOCITIES IN SHOCKED LIQUID DEUTERIUM
}

\author{
N. C. Holmes, W. J. Nellis, and M. Ross \\ Lawrence Livermore National Laboratory, Livermore, CA 94550
}

\begin{abstract}
Recent measurements of shock temperatures and laser-driven Hugoniot measurements of shocked liquid deuterium strongly indicate that molecular dissociation is important above $20 \mathrm{GPa}$. Since the effect of dissociation is small on the Hugoniot pressure up to the $30 \mathrm{GPa}$ limit of conventional impact experiments, other methods must be used to test our understanding of the physics of highly compressed deuterium in this regime. We have recently performed experiments to measure the sound velocity of deuterium which test the isentropic compressibility, a derivative quantity. We used the shock overtake method to measure the shock velocity at 28 GPa. These preliminary data provide support for a recently developed molecular dissociation model.
\end{abstract}

\section{INTRODUCTION}

During the past several years, the properties of shocked liquid deuterium and hydrogen have been the subject of many new experimental studies. The equation of state (EOS) along the principal Hugoniot was determined by Nellis, et al. (1), and theoretical models were developed subsequently. $(2,3)$ Recently, new shock temperature measurements led to the development of a new model, in which the effective molecular dissociation energy decreases with decreasing volume. (4) Extrapolation of those results to pressures above $100 \mathrm{GPa}$ indicated that deuterium would be substantially more compressible in this pressure region than previously thought. More recently, laserdriven experiments were performed to test that prediction, (5) and those results support the predictions of the model in large part. However, the uncertainties in the latter experiments are large. In other experiments, Weir, et al. (6) found evidence for the formation of metallic hydrogen at higher densities and lower temperatures, using quasi-isentropic compression of the liquid.

Measurements of the EOS of the fluid in the pressure range of the most accurate experimental methods (impacts) generally will not provide convincing evidence of small amounts of molecular dissociation, since their effect on the EOS below $30 \mathrm{GPa}$ is small. This suggests the measurement of derivative quantities such as sound velocity or the Grüneisen parameter, both of which should be sensitive to the to the rate of change of compressibility. The sound velocity $c$ of a rarefaction wave is given by

$$
c^{2}=-\left(\frac{\partial P}{\partial \rho}\right)_{S}
$$

where $P$ is pressure, $\rho$ density, and the derivative is at constant entropy $S$. The sound velocity is also related to the Grüneisen parameter through the expression (7)

$c=V\left[\left(\frac{\partial P}{\partial V}\right)_{H}\left[\left(V_{0}-V\right) \frac{\gamma}{2 V}-1\right]+P \frac{\gamma}{2 V}\right]^{1 / 2}$

where the Grüneisen parameter given by

$$
\gamma=V\left(\frac{\partial P}{\partial E}\right)_{V}
$$

can be obtained from shock Hugoniot experiments. Measurements of the sound velocity using the overtake method (8) can provide high accuracy, and an unambiguous test of our understanding of dense, hot, fluid deuterium. 


\section{EXPERIMENTAL}

The shock overtake method exploits the fact that the sound velocity behind a shock is greater than the shock velocity, and thus will overtake it eventually. A target is impacted with a thin impactor; when the impact-generated shock reaches the back surface of the impactor, the pressure drops and a rarefaction wave moves at the sound velocity into the target. If the sound velocities in all layers at high pressure are known, then the time when the overtaking rarefaction reaches the shock provides a direct measure of the sound velocity. For shocked fluids at high temperatures such as deuterium, the intensity of the emitted light varies very strongly with shock velocity or pressure. Thus, observation of the light intensity emitted by the shock front provides a sensitive way of determining the overtake time.

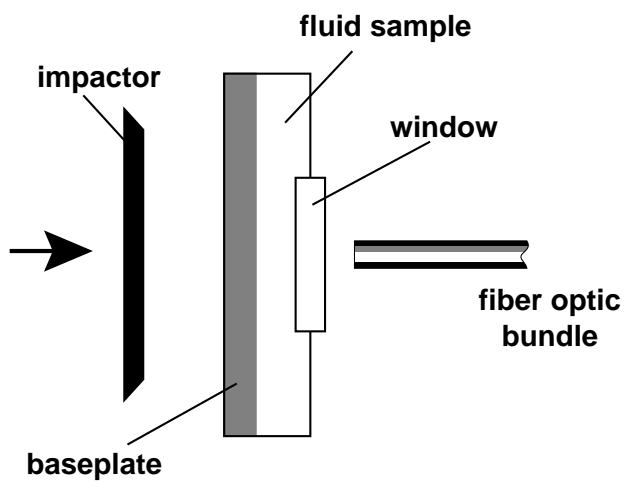

FIGURE 1. Schematic of the optical arrangement for sound velocity experiments. The Ta impactor in embedded in a polycarbonate sabot (not shown), and impacts the Al baseplate, generating a strong shock. Light emitted by the shocked liquid $\mathrm{D}_{2}$ sample is collected by a seven-fiber bundle which is placed near the $\mathrm{Al}_{2} \mathrm{O}_{3}$ window. Not shown are trigger pins and a Pt resistance thermometer placed inside the sample cavity.

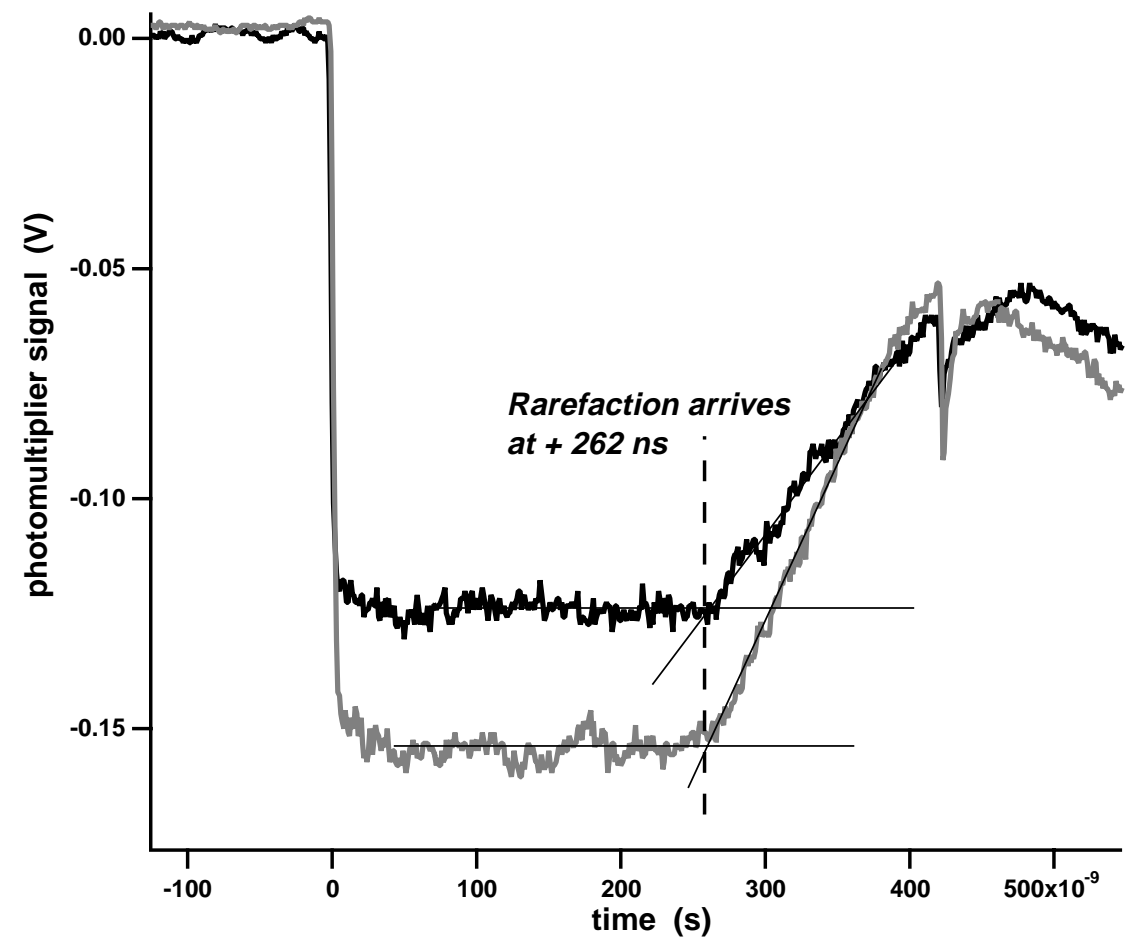

FIGURE 2. Photomultiplier data showing time dependence of light emitted from shocked liquid deuterium at 506 and $700 \mathrm{~nm}$, for the experiments described in the text at a pressure of $28 \mathrm{GPa}$. The regions of relatively constant signal $(0<t<260 \mathrm{~ns})$ indicate steady shock propagation in the $\mathrm{D}_{2}$ sample. The overtaking rarefaction wave decreases the pressure and emitted intensity when it arrives at the shock front. To determine the arrival time, we made lineat fits to the data, and took the intersection as the arrival time. The sharp and brief intensity peak at about $420 \mathrm{~ns}$ is due to shock arrival at the $\mathrm{A} 12 \mathrm{O} 3$ window. 
We used a two-stage light gas gun to accelerate a thin $(0.5 \mathrm{~mm})$ Ta projectile to $7.41 \mathrm{~km} / \mathrm{s}$ in the experiment described here. The design of the cryogenic targets and sample preparation methods for hydrogen experiments were described elsewhere. (1) The projectile was incident on a $1.5 \mathrm{~mm} \mathrm{Al}$ baseplate, and the $\mathrm{D}_{2}$ sample was $6 \mathrm{~mm}$ thick, with an initial temperature of $20 \mathrm{~K}$ and density of $0.171 \mathrm{~g} / \mathrm{cm}^{3}$. Light emitted from the shocked sample was collected by a 7-fiber bundle placed next to an $\mathrm{Al}_{2} \mathrm{O}_{3}$ window in contact with the sample. This system has been described previously $(9,4)$ and is depicted schematically in Fig. 1. Measurement of the steady part of the signal provides a measure of temperature and emissivity of the shocked $\mathrm{D}_{2}$ at the Hugoniot pressure of $28 \mathrm{GPa}$. Since the rise of the light emission occurs when the shock enters the sample (it becomes optically thick in $<1 \mathrm{~ns}$ ), the overtake time can be located easily as shown in Fig. 2.

We used a graphical method to determine the overtake velocity, as depicted in Fig. 3. In the figure, the

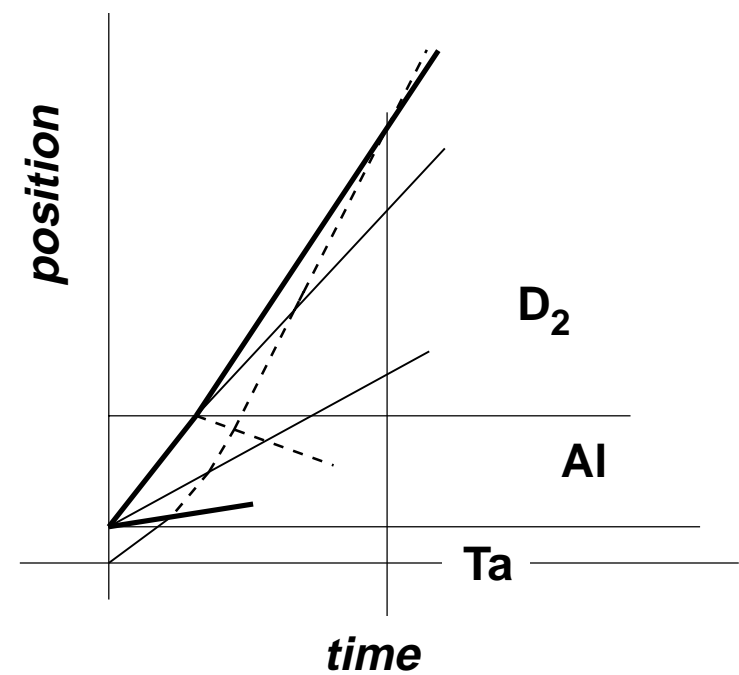

FIGURE 3. Wave interactions and graphical solution for the overtake experiment in liquid $\mathrm{D}_{2}$. Bold lines indicate shock fronts, thin lines the position of material interfaces, and dashed lines the rarefaction waves. Note that the rarefaction which eventually overtakes the $\mathrm{D}_{2}$ shock traverses a region of shocked and released $\mathrm{Al}$

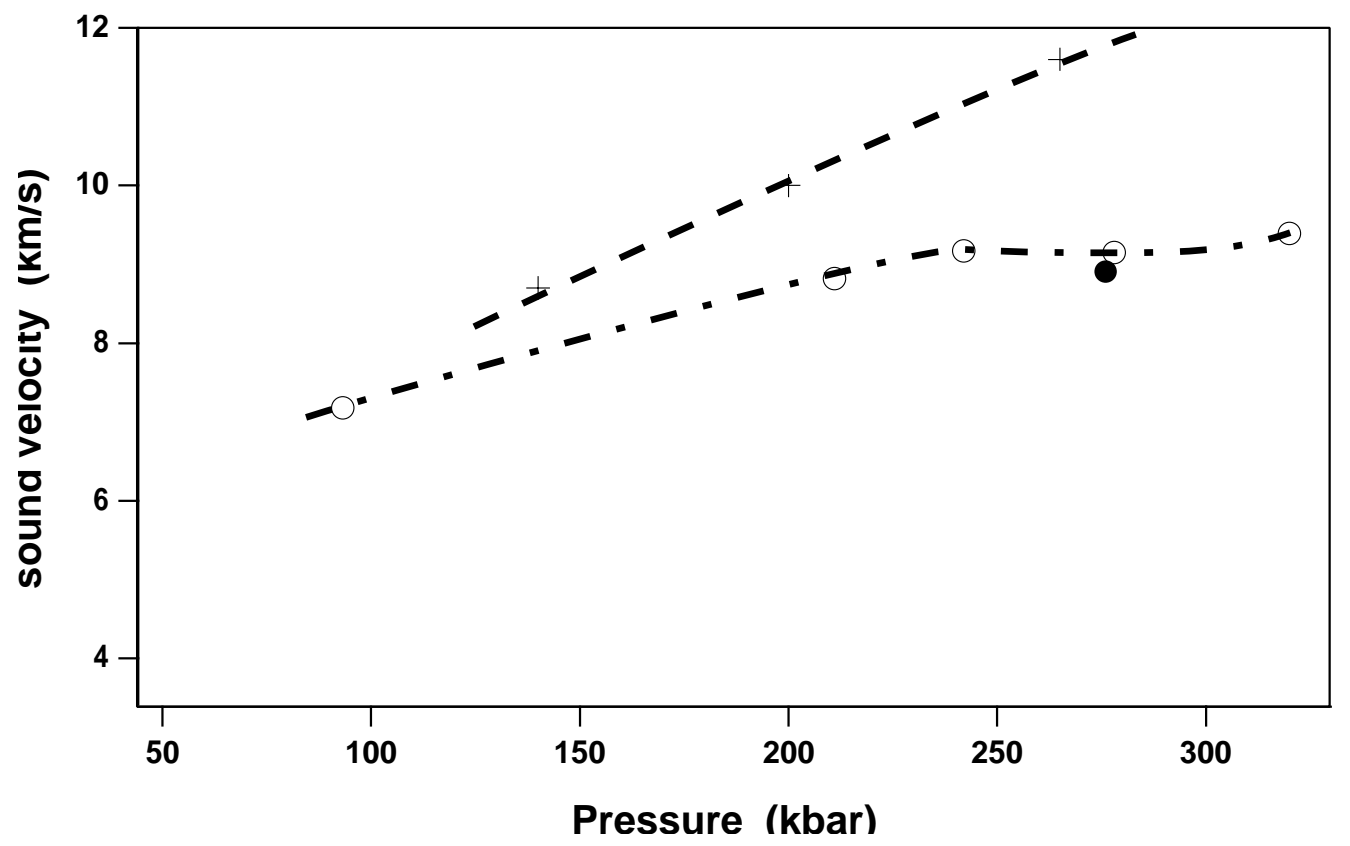

FIGURE 4. Comparison of two calculations of the sound velocity in shocked deuterium vs.shock pressure. The dashed line is calculated from Hugoniot data on liquid $\mathrm{H}_{2}$ and $\mathrm{D}_{2}$, the dot-dash line and open circles from the model presented in Ref 4 . The experimental point at $28 \mathrm{GPa}$ and a compressed density of $0.6 \mathrm{~g} / \mathrm{cm}^{3}$, with a sound velocity of $8.91 \mathrm{~km} / \mathrm{s}$ is depicted by the solid circle. Since this datum is preliminary, the experimental uncertainty has yet to be determined, and no error bars are shown. 
positions of the material interfaces are shown by thin solid lines, the shocks by bold lines, and the leading edge of the rarefaction waves are represented by dashed lines. The sound velocity in shocked Ta at the initial shock pressure of $189 \mathrm{GPa}$ was found by Shaner, et al. (10), and we used unpublished measurements of the sound velocity in shocked $\mathrm{Al}$ at this pressure by Brown. (11) The experimental geometry we used is complicated by a backward rarefaction wave at the $\mathrm{Al} / \mathrm{D}_{2}$ interface. We used a simple Grüneisen model of the Al to calculate the sound velocity in $\mathrm{Al}$ initially shocked to $189 \mathrm{GPa}$ and released to $27.6 \mathrm{GPa}$.

At the conditions of the experiment, the $\mathrm{D}_{2}$ sample density and temperature are $0.653 \mathrm{~g} / \mathrm{cm}^{3}$ and $5500 \mathrm{~K}$, respectively. However, we note that these values are based on a modest extrapolation of the measured Hugoniot. The sound velocity was found to be 8.91 $\mathrm{km} / \mathrm{s}$. Since the sound velocity in the shocked and released $\mathrm{Al}$ is still somewhat uncertain, this value should be viewed as preliminary.

Now let us compare this datum with the predictions of the new model proposed earlier (4), and calculations based on previous experiments. The experiments reported by Nellis, et al. (1) in shocked $\mathrm{H}_{2}$ and $\mathrm{D}_{2}$ allow us to calculate an approximate Grüneisen parameter, since we can determine Eq. (3), and thus sound velocity (Eq. 2). These are plotted in Fig. 4, and show that the present data support the idea of enhanced dissociation of $\mathrm{D}_{2}$ at moderate shock pressures. Clearly, more data are needed over a wider pressure range.

\section{ACKNOWLEDGMENTS}

We are grateful to many for success of this experiment, and wish to credit J. Crawford, K. Stickel, and W. Brocious for two-stage gun operations, and E. F. See, Jr. for target fabrication and characterization. We thank J. M. Brown for the use of his unpublished data for the sound velocities in shocked $\mathrm{Al}$, and $\mathrm{A}$. C. Mitchell for many helpful discussions. This work was supported by the U. S. Department of Energy under contract W-7405-ENG-48.

\section{REFERENCES}

1. W.J. Nellis, A.C. Mitchell, M.van Thiel, G.J. Devine, R.J. Trainor and N. Brown, J. Chem. Phys 79, 1480 (1983).

2. D. Saumon and G. Chabrier, Phys. Rev. A44, 5122 (1991) ; D.

Saumon and G. Chabrier, Phys. Rev. A46, 2084 (1992).

3. M. Ross, F.H.Ree and D.A.Young, J. Chem. Phys. 79, 1487(1983).

4. N. C. Holmes, W. J. Nellis, and M. Ross, Phys. Rev. B52, 15835 (1995).

5. L. B. Da Silva, P. Celliers, G. W. Collins, K. S. Budil, N. C. Holmes, T. W. Barbee, Jr., B. A. Hammel, J. D. Kilkenny, R. J. Wallace, M. Ross, R. Cauble, A. Ng, and G. Chiu, Phys. Rev. Lett. 78, 483 (1997).

6. S. T. Weir, A. C. Mitchell, and W. J. Nellis, Phys. Rev. Lett. 76, 1860 (1996)

7. R. G. McQueen, S. P. Marsh, and J. N. Fritz, J. Geophys. Res. 72, 4999 (1967).

8. R. G. McQueen, J. W. Hopson, and J. N. Fritz, Rev. Sci. Instrum. 53, 245 (1982).

9. N. C. Holmes, Rev. Sci. Instrum. 66, 2615 (1995).

10. J. W. Shaner, J. M. Brown, and R. G. McQueen, in High Pressure in Science and Technology, Homan, MacCrone and Whalley, eds. North-Holland (New Yor,k, 1984) p. 137 ff.

11. J. M. Brown, private communication 